\title{
Numerical Approximation of Microorganisms Hybrid Nanofluid Flow Induced by a Wavy Fluctuating Spinning Disc
}

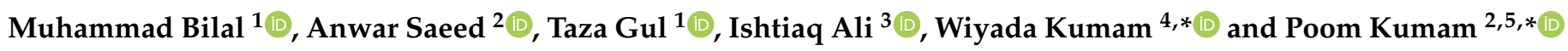 \\ 1 Department of Mathematics, City University of Science and Information Technology, \\ Peshawar 25000, Pakistan; bilalchd345@gmail.com (M.B.); tazagul@cusit.edu.pk (T.G.) \\ 2 Center of Excellence in Theoretical and Computational Science (TaCS-CoE), Faculty of Science, \\ King Mongkut's University of Technology Thonburi (KMUTT), 126 Pracha Uthit Rd., Bang Mod, Thung Khru, \\ Bangkok 10140, Thailand; anwarsaeed769@gmail.com \\ 3 Department of Mathematics and Statistics, College of Science, King Faisal University, P.O. Box 400, \\ Hafouf 31982, Saudi Arabia; iamirzada@kfu.edu.sa \\ 4 Applied Mathematics for Science and Engineering Research Unit (AMSERU), Program in Applied Statistics, \\ Department of Mathematics and Computer Science, Faculty of Science and Technology, \\ Rajamangala University of Technology Thanyaburi, Thanyaburi, Pathumthani 12110, Thailand \\ 5 Department of Medical Research, China Medical University Hospital, China Medical University, \\ Taichung 40402, Taiwan \\ * Correspondence: wiyada.kum@rmutt.ac.th (W.K.); poom.kum@kmutt.ac.th (P.K.)
}

Citation: Bilal, M.; Saeed, A.; Gul, T.; Ali, I.; Kumam, W.; Kumam, P.

Numerical Approximation of

Microorganisms Hybrid Nanofluid Flow Induced by a Wavy Fluctuating Spinning Disc. Coatings 2021, 11, 1032 https://doi.org/10.3390/

coatings11091032

Academic Editor: Mikhail Sheremet

Received: 10 August 2021

Accepted: 25 August 2021

Published: 27 August 2021

Publisher's Note: MDPI stays neutral with regard to jurisdictional claims in published maps and institutional affiliations.

Copyright: (C) 2021 by the authors Licensee MDPI, Basel, Switzerland. This article is an open access article distributed under the terms and conditions of the Creative Commons Attribution (CC BY) license (https:// creativecommons.org/licenses/by/ $4.0 /)$.

\begin{abstract}
The analysis explored a numerical simulation of microorganisms, carbon nanotubes (CNTs) and ferric oxide water-based hybrid nanofluid flow induced by a wavy fluctuating spinning disc with energy propagation. In the presence of CNTs and magnetic nanoparticulates, the nanofluid is synthesized. The exceptional tensile strength, flexibility, and electrical and thermal conductivity of carbon nanotubes and iron nanoparticles have been extensively reported. The motive of the proposed analysis is to optimize thermal energy conveyance efficiency for a spectrum of industrial and biomedical applications. The phenomena have been expressed as a system of partial differential equations (PDEs) which contain the momentum, energy, concentration, and motile microorganism equations. The modeled equations have been diminished to the dimensionless system of nonlinear ODEs through a similarity framework. The Matlab built-in package boundary value solver has been utilized to solve the obtained system of ODEs. The findings are compared to the PCM technique for validity purposes. The results are illustrated graphically and discussed. The layout of a rotating disc has a positive effect on energy transition and velocity profile. The irregular rotating surface increases energy progression up to $15 \%$ relative to a smooth surface. The accumulation of nanocomposites (CNTs and magnetic nanoparticles) significantly enhanced the thermal capabilities of the liquid medium. When operating with a low distribution, it is more impactful.
\end{abstract}

Keywords: hybrid nanofluid; wavy spinning disk; CNTs; gyrotactic microorganism; MHD; fluctuating disk

\section{Introduction}

The study of hybrid nanofluid flow over a rotating disc with heat and mass transition characteristics has been heavily reported due to its remarkable contribution to modern technologies and industrial requirements. Electric power generating systems, aerodynamics engineering, co-rotating machinery, rotating equipment, chemical reaction, the geothermal sector, and computer processing are some of its well-known applications [1] Lv et al. [2] investigated the effects of magnetic fields and Hall the current on nanofluid flow over a revolving disk. Their intention was to accelerate heat propagation rates for engineering applications. The observations indicated that the mutation of CNTs in water is more beneficial than other nanomaterials due to its carbon-carbon bonding. Li et al. [3] employed FDE12 and the bvp4c package to perform fractional assessments for Darcy hybrid 
nanofluid flow over a permeable rotational disc. At the rotating disk's edge, concentration and temperature slip conditions were used. Shuaib et al. [4] demonstrated the nature of an ionic transition boundary layer flow over a spinning disc using a numerical model. The Nernst-Planck, Poisson's, and the classic Navier Stokes equations are approximated using the PCM and bvp4c schemes for this purpose. Shuaib et al. $[5,6]$ studied the flow and heat transport of 3D incompressible viscous fluids over a stretchy, rough rotating disc. A variable magnetic flux and heat transference are used to control a varying thickness of the fluid. By the action of the no flatness factor, the heat transmission process was examined while being reduced. Hafeez et al. [7] operated with a revolving disc and a magnetized flow of an Oldroyd-B fluid. The thermal energy and fluid velocity profile are observed to decrease as the time relaxation parameter is increased. Liang et al. [8,9] suggested a capillary model, as well as a fractal theory of permeable media, to measure the efficient electrolyte permeability in porous media while taking into account the electrical double layer effects.

Hybrid nanofluids are a new type of fluid that operate well in energy transmission when compared to traditional fluids such as oil, water, and single nanomaterials such as nanofluids and ethylene glycol. Hybrid nanofluids are effective in freezing where the heating rate is high, and they can be used in a wide range of thermal activities. Hybrid nanofluids are made by distributing two different types of NPs in a base fluid [10]. Solar energy, as well as heating, refrigeration, ventilation, heat exchangers, heat pipes, air conditioning, coolant in machines and manufacturing, the automotive industry, electronic cooling, generator cooling, nuclear system cooling, transformer cooling, ships, and biomedicine are just a few of the application domains of hybrid nanofluids. In the present work, we consider CNTs and ferric oxide nanoparticles in the base fluid water. Because graphitic walls are chemically inert, the functionalization of graphene and CNTs is frequently required in engineering and biological activities [11,12]. The major problem for any nanofluid that will be used in thermal management operations is its long-term stability. For 30 days, the distribution stabilities of carbon-based and metallic oxides nanofluids were monitored, and the findings revealed that the nanofluids had a greater dispersibility in an aqueous environment with extremely low sedimentation [13]. A viscous dissipating convective flow of CNT's hybrid nanofluid across a horizontally moving thin needle is addressed by Gul et al. [14]. The utilization of $\mathrm{CNT}+\mathrm{Fe}_{3} \mathrm{O}_{4} / \mathrm{H}_{2} \mathrm{O}$ for the activation of thermophysical features of carrier fluids coupled with magnetic nanoparticles has been discovered to be more prevalent. Tassaddiq et al. [15] developed an impermeable infinite spinning disc with an incompressible hybrid nanofluid flow. To thoroughly analyze the positive spin of nanofluid flow, the effects of magnetism have been imposed. The main objective was to improve our comprehension of energy consumption in the industrial and technical domains. Fluid energy and velocity are estimated by measuring disc rotation velocity. Polymer coatings or the insertion of nanostructures can be used to reduce the negative effects of electroosmotic flow [16,17]. Bilal et al. [18] employed an inclined extensible cylinder to evaluate the Darcy Forchhemier flow of a hybrid nanofluid including CNTs and iron oxide $\mathrm{Fe}_{3} \mathrm{O}_{4}$ nanocrystals. The findings suggest that hybrid nanofluid is the most effective method for improving heat transmission and can also be utilised for cooling. The consequences of a magnetic field on a thin liquid sheet of water-based Ferrium oxide and CNT hybrid nanofluids was evaluated across an extending cylinder by Gul et al. [19]. In carrier fluid water, iron oxide and CNTs have been employed as nanoparticulates. The scattering of $\mathrm{Fe}_{3} \mathrm{O}_{4}$ and CNTs in the base fluid considerably improved the heat transference mechanism. Ghalandari et al. [20] provided a review paper on his work on the thermal characteristics of nanofluids, including carbon nanotubes. It was stated that nanofluids containing carbon nanotubes increase the exergy and energy execution of solar systems.

Low-density microorganisms float above the surface of liquids due to chaotic situations and volatility, a process known as bioconvection. Swimming microorganisms, such as microalgae, are more capable of spreading over the higher liquid layer, which is 
responsible for the unsteady higher component, leading to a greater density of stratification. Such nanostructured materials' mobility is independent of motile microbe locomotion, and the mutual interaction of nanofluids and bioconvection appears to be crucial for nanofluidic equipment [21]. Khan et al. [22] examined the unsteady viscosity and changing thermal repercussions in non-Newtonian nanoliquid over a regularly moving surface to investigate bioconvection. Walter's B liquid is used to evaluate the rheological effects of such fluid. Kotha et al. [23] exploited heat absorption or generation to study the 2D magnetohydrodynamic flow with energy and mass transit processes of nanofluid comprising gyrotactic microorganisms over a lateral plate. The outcomes asserted that the motile microorganism transit ratios along with velocity gradients are reduced across the fluid medium as a result of the magnetic flux on energy and mass propagation.

The goal of this research is to further elaborate a concept proposed in Ref. [24] by observing the effects of two distinct nanoparticles, ferric oxide and CNT's water-based hybrid nanofluids over a fluctuating wavy rotating disc. This study explored the consequences of a magnetic field and nanocomposites on the hybrid nanoliquid flow in order to optimize the thermal conductivity of a water over a spinning surface. The concentration profile and gyrotactic microorganisms enhanced the novelty of the present study. Matlab's package boundary value solver (bvp4c) is used to address the constructed equations computationally, and the findings are certified and contrasted against the parametric continuation process $(\mathrm{PCM})$. This revealed the best settlement.

\section{Mathematical Formulation}

We have considered an unsteady 3D flow of CNTs and ferric oxide water-based hybrid nanofluid flow over a wavy fluctuating moving rotating disk (See Figure 1).

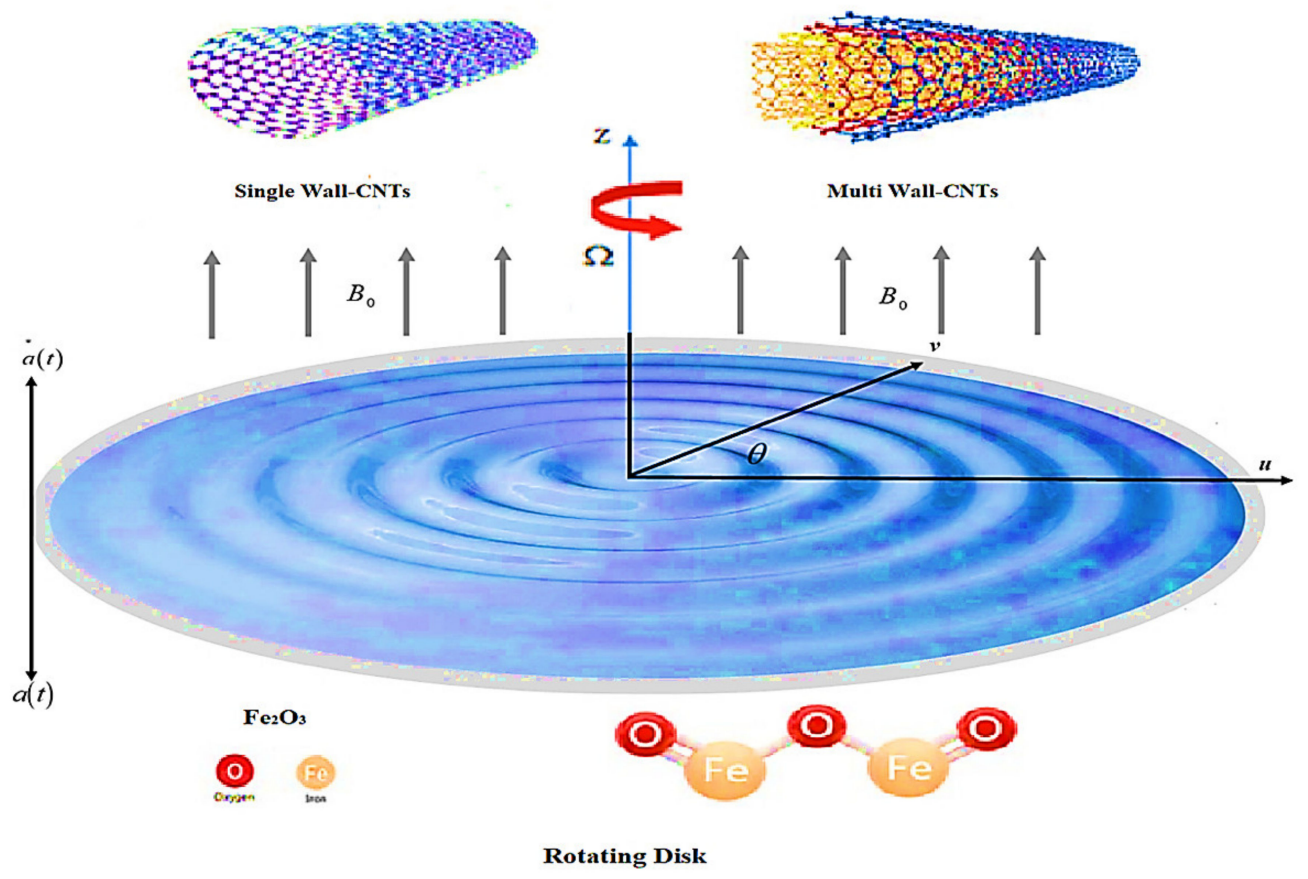

Figure 1. The physical insights of fluid flow over a rotating disk.

The disk was initially kept at $a(0)=h$. But then with the velocity $\omega=a(t)$ the disk moved to vertical distance $Z=a(t)$. The buoyancy influence was assumed to be negligible. The disk spun with angular velocity $\Omega(t)$ at $z$-axis. It has been supposed that the CNTs and iron oxide nano particulates are scattered uniformly. The magnetic force of constant 
magnitude is consistently applied with $\vec{B}=\sqrt{B_{r} \overrightarrow{e_{r}}+B_{\theta}} \overrightarrow{e_{\theta}}$ and $B=\sqrt{B_{r}^{2}+B_{\theta}^{2}}$ respectively. Based on the above assumption, the modeled equations are defined as [25]:

$$
\begin{gathered}
\frac{\partial u}{\partial r}+\frac{\partial w}{\partial z}+\frac{u}{r}=0 \\
\rho_{h n f}\left(\frac{\partial u}{\partial t}+u \frac{\partial u}{\partial r}+w \frac{\partial u}{\partial z}-\frac{v^{2}}{r}\right)=-\frac{\partial p}{\partial r}+\mu_{h n f}\left(\frac{\partial^{2} u}{\partial r^{2}}+\frac{\partial^{2} u}{\partial z^{2}}-\frac{u}{r^{2}}+\frac{1}{r} \frac{\partial u}{\partial r}\right)+F_{r} \\
\rho_{h n f}\left(\frac{\partial v}{\partial t}+u \frac{\partial v}{\partial r}+w \frac{\partial v}{\partial z}-\frac{u v}{r}\right)=\mu_{h n f}\left(\frac{\partial^{2} v}{\partial r^{2}}+\frac{\partial^{2} v}{\partial z^{2}}-\frac{v}{r^{2}}+\frac{1}{r} \frac{\partial v}{\partial r}\right) \\
\rho_{h n f}\left(\frac{\partial w}{\partial t}+u \frac{\partial w}{\partial r}+w \frac{\partial w}{\partial z}\right)=-\frac{\partial p}{\partial z}+\mu_{h n f}\left(\frac{\partial^{2} w}{\partial r^{2}}+\frac{\partial^{2} w}{\partial z^{2}}+\frac{1}{r} \frac{\partial w}{\partial r}\right)+F_{\theta} \\
\left(\frac{\partial T}{\partial t}+u \frac{\partial T}{\partial r}+w \frac{\partial T}{\partial z}\right)=\frac{k}{\left(\rho C_{p}\right)_{h n f}}\left(\frac{\partial^{2} T}{\partial r^{2}}+\frac{1}{r} \frac{\partial T}{\partial r}+\frac{\partial^{2} T}{\partial z^{2}}\right) \\
\left(\frac{\partial C}{\partial t}+u \frac{\partial C}{\partial r}+w \frac{\partial C}{\partial z}\right)=D_{h n f}\left(\frac{\partial^{2} C}{\partial r^{2}}+\frac{1}{r} \frac{\partial C}{\partial r}+\frac{\partial^{2} C}{\partial z^{2}}\right) \\
\left(\frac{\partial N}{\partial t}+w \frac{\partial N}{\partial z}+\widetilde{w} \frac{\partial N}{\partial z}+N \frac{\partial N}{\partial z}\right)=D_{n}\left(\frac{\partial^{2} N}{\partial z^{2}}\right)
\end{gathered}
$$

Here $F_{r}$ and $F_{\theta}$ are the body forces along $x$ and $z$ direction, which can be indicated as [26]:

$$
\begin{gathered}
F_{r}=\frac{H a^{2} \mu_{h n f}}{R^{2}}\left(v \sin \theta \cos \theta-u \sin ^{2} \theta\right), \\
F_{\theta}=\frac{H a^{2} \mu_{h n f}}{R^{2}}\left(u \sin \theta \cos \theta-v \sin ^{2} \theta\right),
\end{gathered}
$$

where $u, v, w$ are the velocity component, Ha is $L B_{0} \sqrt{\sigma / \mu}$, in which $B_{0}$ and $\theta$ is the magnitude and direction of magnetic field.

The initial and boundary conditions are:

$$
\left.\begin{array}{l}
u=0, v=r \Omega_{0}(t), w=\beta^{*}(t), T=T_{0}, C=C_{0}, \widetilde{N}=\widetilde{N}_{w}, \text { at } z=0 \\
u \rightarrow 0, v \rightarrow 0, w \rightarrow 0, T \rightarrow T_{\infty}, C \rightarrow C_{\infty}, \widetilde{N} \rightarrow \widetilde{N}_{\infty}, \text { at } z \rightarrow \infty .
\end{array}\right\}
$$

\section{Thermophysical Properties of Nanofluid}

The thermal physical properties of CNTs and iron oxide hybrid nanofluid can be calculated as [27]:

Here, $\phi_{1}$ and $\phi_{2}$ is the volume friction of CNTs and ferric oxide, $k_{h n f}, k_{f}$ is the thermal conductivity, $\rho_{f}$ is the density, $\left(C_{p}\right)_{M S}$ and $\rho_{M S}$ is the specific heat capacities.

Tables 1 and 2 illustrate the model and thermophysical properties of base fluid, ferric oxide nanoparticles and carbon nanotubes of both types, single and multi-wall, respectively. 
Table 1. The thermophysical properties of base fluid and hybrid nanofluid.

\begin{tabular}{cc}
\hline$v_{h n f}=\frac{\mu_{h n f}}{\rho_{h n f}}$, & $\mu_{h n f}=\frac{\mu_{f}}{\left(1-\phi_{1}\right)^{5 / 2}\left(1-\phi_{2}\right)^{5 / 2},}$ \\
\hline$\frac{(\rho)_{h n f}}{(\rho)_{f}}=\left\{1-\left(1-\frac{(\rho) M s}{(\rho)_{f}}\right) \phi_{1}\right\}\left(1-\phi_{2}\right)+\frac{(\rho)_{C N T}}{(\rho)_{f}} \phi_{2}$, & $\frac{\left(\rho C_{p}\right)_{h n f}}{\left(\rho C_{p}\right)_{f}}=\left\{1-\left(1-\frac{\left(\rho C_{p}\right) M s}{\left(\rho C_{p}\right)_{f}}\right) \phi_{1}\right\}\left(1-\phi_{2}\right)+\frac{\left(\rho C_{p}\right)_{C N T}}{\left(\rho C_{p}\right)_{f}} \phi_{2}$, \\
$\frac{k_{h n f}}{k_{b f}}=\frac{1-\phi_{2}+2 \phi_{2} \frac{k_{C N T}}{\left(k_{C N T}-k_{b f}\right)}-\ln \frac{{ }^{{ }^{C} C N T+k_{b f}}}{2 k_{b f}}}{1-\phi_{2}+2 \phi_{2} \frac{k_{b f}}{\left(k_{C N T}-k_{b f}\right)}-\ln \frac{{ }{ }^{C N T}+k_{b f}}{2 k_{b f}}}$, & $\frac{k_{b f}}{k_{f}}=\frac{k_{M S}+(\mathrm{m}-1)_{k f}-(\mathrm{m}-1) \phi_{1}\left(\mathrm{k}_{f}-\mathrm{k}_{M S}\right)}{k_{M S}+(\mathrm{m}-1)_{k f}-\phi_{1}\left(\mathrm{k}_{f}-\mathrm{k}_{M S}\right)}$, \\
\hline
\end{tabular}

Table 2. The numerical properties of water, $\mathrm{CNTs}$ and $\mathrm{Fe}_{3} \mathrm{O}_{4}$.

\begin{tabular}{cccc}
\hline $\begin{array}{c}\text { Base } \\
\text { Fluid/Nanoparticles }\end{array}$ & $\boldsymbol{\rho}\left(\mathbf{k g} / \mathbf{m}^{3}\right)$ & $C_{p}(\mathbf{j} / \mathbf{k g K})$ & $k(\mathbf{W} / \mathbf{m K})$ \\
\hline Water & 997.1 & 4179 & 0.613 \\
$\mathrm{Fe}_{3} \mathrm{O}_{4}$ & 5200 & 670 & 6 \\
$\mathrm{SWCNTs}$ & 2600 & 425 & 6600 \\
MWCNTs & 1600 & 796 & 300 \\
\hline
\end{tabular}

\section{Karman's Approach}

We use the following similarity framework to reduce Equations (1)-(8) to the system of ODEs [28]:

$$
\left.\begin{array}{l}
u=\frac{r v}{a^{2}(t)} f(\eta), v=\frac{r v}{a^{2}(t)} g(\eta), w=\frac{v}{a(t)} h(\eta), p=\frac{p v^{2}}{a^{2}(t)} p(\eta), C=C_{\infty}+\Delta C_{\infty}, \\
\eta=\frac{Z}{a(t)}-1, \quad T=T_{\infty}+\Delta T_{\infty}, \eta_{Z}=\frac{1}{a(t)}, \eta_{t}=\frac{-a(t)}{a(t)}(\eta+1), \Psi(\eta)=\frac{N-N_{0}}{N_{1}-N_{0}} .
\end{array}\right\}
$$

As a result of Equation (14), we get the following:

$$
\begin{gathered}
f^{\prime \prime}=\frac{\rho_{h n f}}{\mu_{h n f}}\left(h f^{\prime}+f^{2}-g^{2}-S \frac{(\eta+1) f^{\prime}}{2}+f\right)+A \omega\left(g \sin \theta \cos \theta-f \sin ^{2} \theta\right), \\
g^{\prime \prime}=\frac{\rho_{h n f}}{\mu_{h n f}}\left(h g^{\prime}+2 f g-S\left(\frac{(\eta+1) g^{\prime}}{2}-g\right)\right), \\
h^{\prime \prime}=\frac{\rho_{h n f}}{\mu_{h n f}}\left(h h^{\prime}-S \frac{(\eta+1) h^{\prime}}{2}+h^{\prime}\right)-A \omega\left(f \sin \theta \cos \theta-g \sin ^{2} \theta\right), \\
\Theta^{\prime \prime}=\rho_{h n f}\left(\operatorname{Prh} \Theta^{\prime}-\operatorname{Pr} S\left(\frac{(\eta+1) \Theta^{\prime}}{2}+\gamma \Theta\right)\right), \\
\Phi^{\prime \prime}=S c h \Phi^{\prime}-S c S\left(\frac{\eta+1}{2}\right) \Phi^{\prime}, \\
\Psi^{\prime \prime}=S c \Psi^{\prime}(2 f-\eta \beta)+\operatorname{Pe}\left(\Psi^{\prime} \Phi^{\prime}+\Psi \Phi^{\prime \prime}+N_{\delta} \Phi^{\prime \prime}\right) .
\end{gathered}
$$

The transform conditions are:

$$
\left.\begin{array}{l}
f(0)=0, h(0)=\beta \frac{S}{2}, g(0)=\omega, \Theta(0)=1, \Phi(0)=1, \Psi(0)=1 \text { at } \eta=0, \\
f(\eta) \rightarrow 0, g(\eta) \rightarrow 0, h(\eta) \rightarrow 0, \Theta(\eta) \rightarrow 0, \Phi(\eta) \rightarrow 0, \Psi(\eta) \rightarrow 0 \text { as } \eta \rightarrow \infty .
\end{array}\right\}
$$

where $P e$ is the bioconvective Peclet number, $\omega$ is the disk's rotation, $S$ is fluctuating parameter of the disk, $N_{\delta}$ is the ratio of distributions of the microorganisms over the disk and $\gamma$ is the thermal energy ratio parameter, which can be expressed as [28]:

$$
P e=\frac{b W c}{D n}, S=2 \frac{a^{*}(t) a(t)}{v}, \omega=2 \frac{a^{2}(t) \Omega(t)}{v}, N_{\delta}=\left(\frac{N_{0}}{N_{0}-N_{1}}\right), \gamma=\frac{1}{2} \frac{a(t) T}{a^{*}(t) \Delta T} .
$$


The skin friction, Nusselt number and Sherwood number are:

$$
C_{f}=\frac{\sqrt{\tau_{w r}^{2}+\tau_{w \phi}^{2}}}{(\Omega r)^{2} \rho_{f}}, \quad N u=\frac{q_{w} r}{\left(T_{w}-T_{\infty}\right) k_{f}}, \quad S h=\frac{j_{w} r}{\left(C_{w}-C_{\infty}\right) D_{f}},
$$

where,

$$
\begin{aligned}
& \tau_{w r}=\left[\mu_{h n f}\left(\frac{d u}{d z}+\frac{d w}{d \phi}\right)\right]_{z=0}{ }^{\prime} \tau_{w \phi}=\left[\mu_{h n f}\left(\frac{d v}{d z}+\frac{1}{r} \frac{d w}{d \phi}\right)\right]_{z=0}{ }^{\prime} q_{w}=-\frac{k_{h n f}}{k_{f}}\left(\frac{d T}{d z}\right)_{z=0^{\prime}} \\
& j_{w}=-D_{h n f}\left(\frac{d C}{d z}\right)_{z=0} .
\end{aligned}
$$

The dimensionless form of Equation (22) is:

$$
\operatorname{Re}^{\frac{1}{2}} C_{f}=\frac{\sqrt{\left(F^{\prime}(0)\right)^{2}+\left(G^{\prime}(0)\right)^{2}}}{\left(1-\phi_{1}\right)^{2.5}\left(1-\phi_{2}\right)^{2.5}}, \operatorname{Re}^{\frac{-1}{2}} N u=-\frac{k_{h n f}}{k_{f}} \Theta^{\prime}(0), \operatorname{Re}^{\frac{-1}{2}} S h=-\Phi^{\prime}(0), \operatorname{Re}=\frac{\Omega r^{2}}{v_{f}} .
$$

\section{Numerical Solution}

We introduced the following similarity variables to reduce the system of ODE (12-17) to the first order system of ODEs and their boundary conditions (18):

$$
\left.\begin{array}{c}
\chi_{1}(\eta)=f(\eta), \quad \chi_{2}=f^{\prime}(\eta), \quad \chi_{3}=g(\eta), \quad \chi_{4}=g^{\prime}(\eta), \quad \chi_{5}=h(\eta), \quad \chi_{6}=h^{\prime}(\eta), \\
\chi_{7}(\eta)=\theta(\eta), \quad \chi_{8}=\theta^{\prime}(\eta), \quad \chi_{9}=\Phi(\eta), \quad \chi_{10}=\Phi^{\prime}(\eta), \quad \chi_{11}=\Psi(\eta), \chi_{12}=\Psi^{\prime}(\eta) .
\end{array}\right\}
$$

Using Equation (25) into the BVP (14-19) and (20), we get:

$$
\begin{gathered}
\chi_{2}{ }^{\prime}=\frac{\rho_{h n f}}{\mu_{h n f}}\left(\left(\chi_{5}-S \frac{(\eta+1)}{2}\right) \chi_{2}+\left(\chi_{1}\right)^{2}-\left(\chi_{3}\right)^{2}-S \chi_{1}\right)+A \omega\left(\chi_{3} \sin \theta \cos \theta-\chi_{1} \sin ^{2} \theta\right), \\
\chi_{4}{ }^{\prime}=\frac{\rho_{h n f}}{\mu_{h n f}}\left(\left(\chi_{5}-S \frac{(\eta+1)}{2}\right) \chi_{4}+2 \chi_{1} \chi_{3}-S \chi_{3}\right), \\
\chi_{6}{ }^{\prime}=\frac{\rho_{h n f}}{\mu_{h n f}}\left(\left(\chi_{5}-S \frac{(\eta+1)}{2}+1\right) \chi_{6}\right)-A \omega\left(\chi_{1} \sin \theta \cos \theta-\chi_{3} \sin ^{2} \theta\right) \\
\chi_{8}{ }^{\prime}=\rho_{h n f}\left(\left(\operatorname{Pr} \chi_{5}-\operatorname{Pr} S \frac{(\eta+1)}{2}\right) \chi_{8}-S \chi_{7} \gamma\right), \\
\chi_{10}^{\prime}=S c S \chi_{10}-S c S\left(\frac{(\eta+1) \chi_{10}}{2}+\lambda \chi_{7}\right), \\
\chi_{12}^{\prime}=S c \chi_{12}\left(2 \chi_{1}-\eta \beta\right)+\operatorname{Pe}\left(\chi_{12} \chi_{10}+\chi_{11} \chi_{10}^{\prime}{ }_{10}+N_{\delta} \chi_{10}^{\prime}\right),
\end{gathered}
$$

the reduced boundary conditions are:

$$
\left.\begin{array}{c}
\chi_{1}=0, \quad \chi_{3}=\omega, \quad \chi_{5}=\beta \frac{S}{2}, \chi_{7}=1, \chi_{9}=1, \chi_{11}=1 \text { at } \eta=0, \\
\chi_{1} \rightarrow 0, \quad \chi_{3} \rightarrow 0, \chi_{5} \rightarrow 0, \chi_{7} \rightarrow 0, \chi_{9} \rightarrow 0, \chi_{11} \rightarrow 0 \text { at } \eta \rightarrow \infty .
\end{array}\right\}
$$

The well-known bvp4c technique was used for the solution of the problem.

\section{Results and Discussion}

The numerical outputs of the system of DEs are calculated by using the MATLAB built-in package boundary value solver (bvp4c) technique. The following observations have been made:

\subsection{Axial Velocity Profile}

Figure 2a-c elaborated the axial velocity profile $f(\eta)$ versus volume friction parameter of CNTs $\phi_{1}$, volume friction parameter of ferric oxide $\phi_{2}$ and disk fluctuation parameter, $S$ respectively. The specific heat capacity of water is much higher than both type of CNTs, single (SWCNTs) as well as multi-wall (MWCNTs), and magnetic nanoparticles $\phi_{2}=\phi_{\mathrm{Fe}_{2} \mathrm{O}_{3}}$, 
so the inclusion of such nanomaterials in base fluid reduces its average capability of heat absorption, which results in the enhancement of fluid velocity, as shown in Figure 2a,b. The upward/downward fluctuation of the spinning disk encourages the fluid particles to move fast, which elevates the axial velocity of fluid as displayed in Figure 2c.

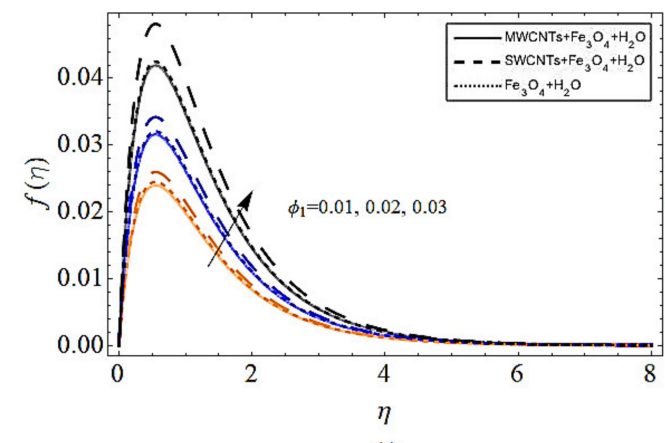

(a)

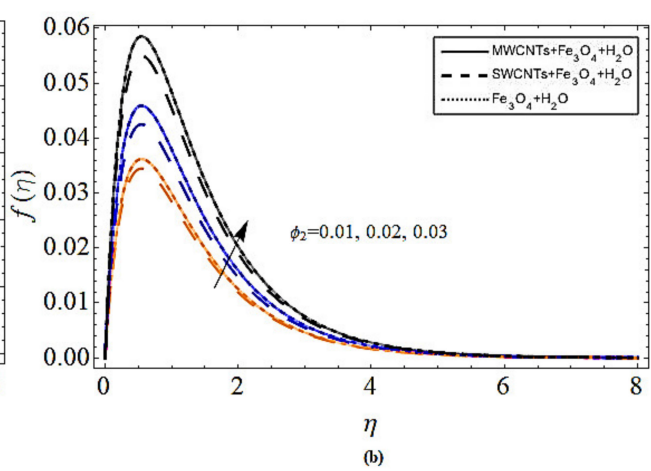

(b)

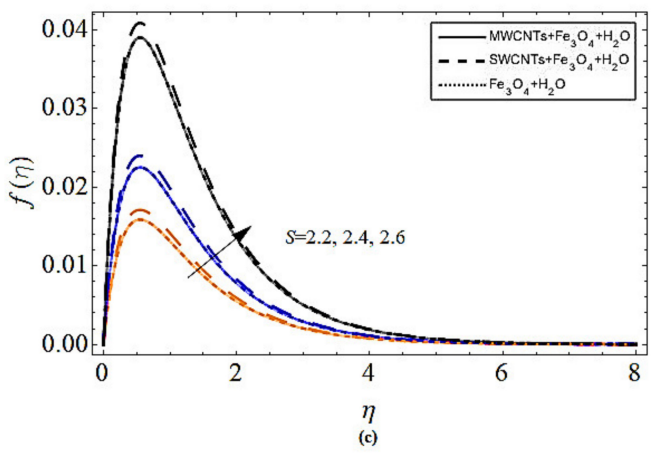

Figure 2. The behavior of axial velocity $f(\eta)$ versus (a) volume friction parameter of CNTs $\phi_{1}$ (b) volume friction parameter of ferric oxide $\phi_{2}$ (c) disk fluctuation parameter $S$.

\subsection{Radial Velocity Profile}

Figure 2a-c elaborated the radial velocity profile $h(\eta)$ behavior versus injection $+\beta$, suction parameter $-\beta$ and disk rotation parameter $\omega$ effects, respectively. Both the suction and injection effect over the surface of the spinning disk produces resistance to the flow field, which causes the reduction of radial velocity $h(\eta)$ profile as revealed through Figure $3 a, b$. The increment in disk rotation rate also excited the fluid particles during rotating, which resulted in a rise of the radial velocity of fluid over a wavy surface, as in Figure 3c.

\subsection{Temperature Profile}

Figure 4a-d illustrated the behavior of energy profile $\Theta(\eta)$ versus thermal energy ratio parameter $\gamma$, Prandtl number $P r$, volume friction parameter of CNTs $\phi_{1}$ and volume friction parameter of ferric oxide $\phi_{2}$ respectively. The thermal energy transmission rate declines with the impact of both thermal energy ratio parameter $\gamma$ and Prandtl number $\operatorname{Pr}$ as illustrated in Figure 4a,b. Higher Prandtl fluid has always less thermal diffusivity, which is why the fluid temperature propagation reduces with the Prandtl effect as specified through Figure $4 \mathrm{~b}$. The specific heat capacity of water is much higher than CNTs $\phi_{1}=\phi_{C N T}$ and magnetic nanoparticles $\phi_{2}=\phi_{\mathrm{Fe}_{2} \mathrm{O}_{3}}$, so the dispersion of such nanomaterials in base fluid reduces its average capability of heat absorption, which results in the enhancement of fluid temperature as shown in Figure 4c,d. 


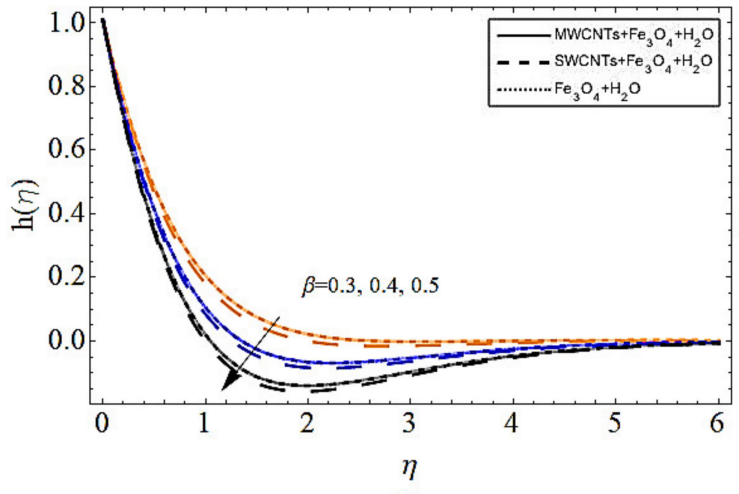

(a)

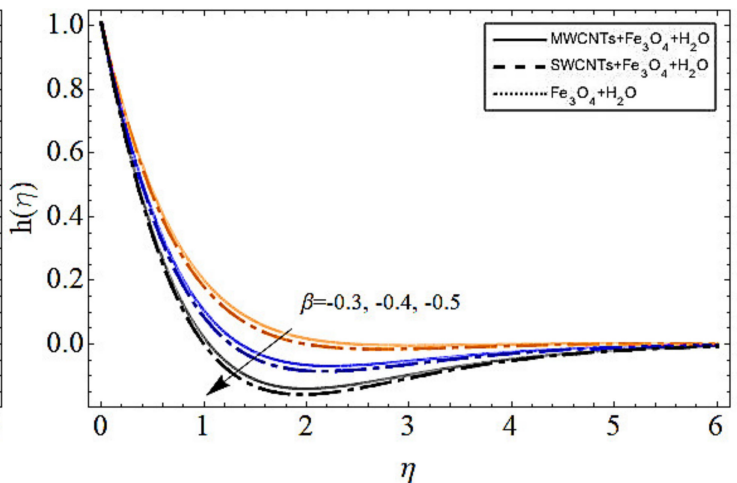

(b)

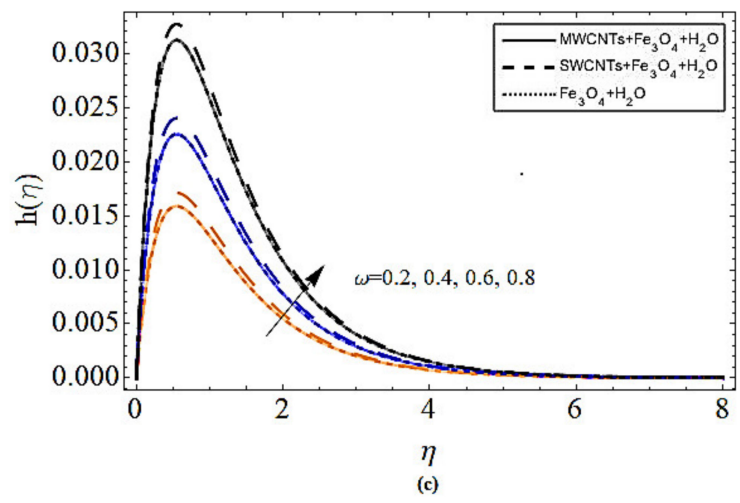

Figure 3. The behavior of radial velocity $h(\eta)$ versus (a) injection parameter $+\beta(\mathbf{b})$ suction parameter $-\beta$ (c) disk rotation parameter $\omega$.

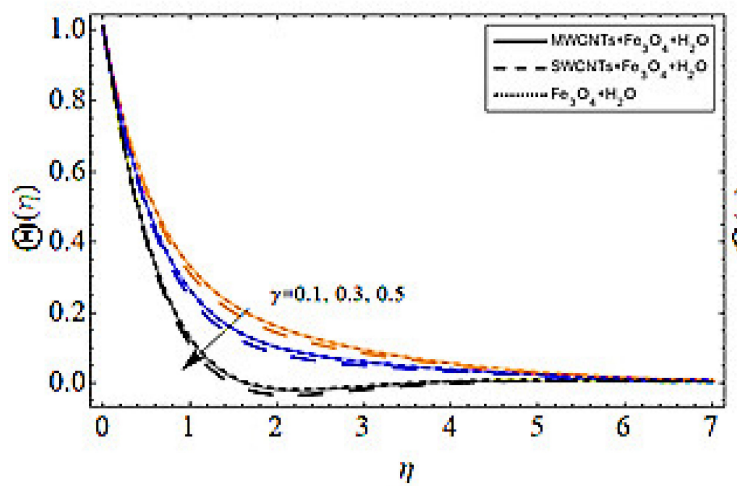

(a)

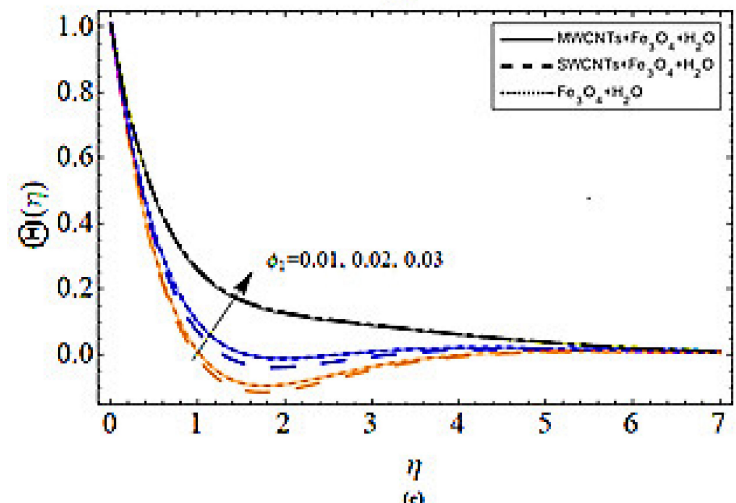

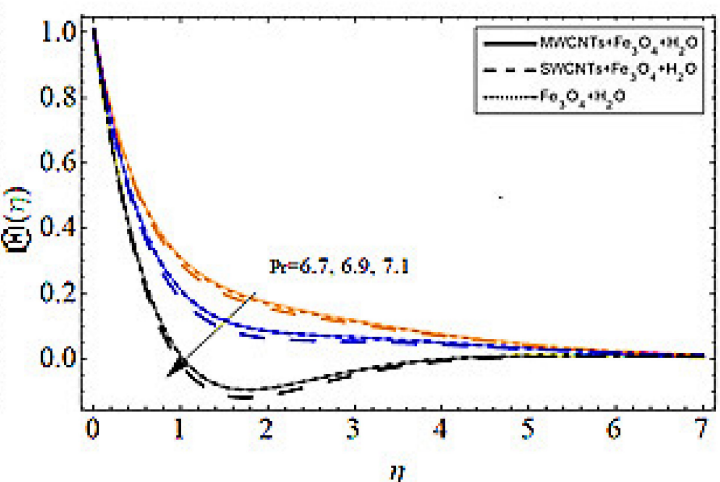

(b)

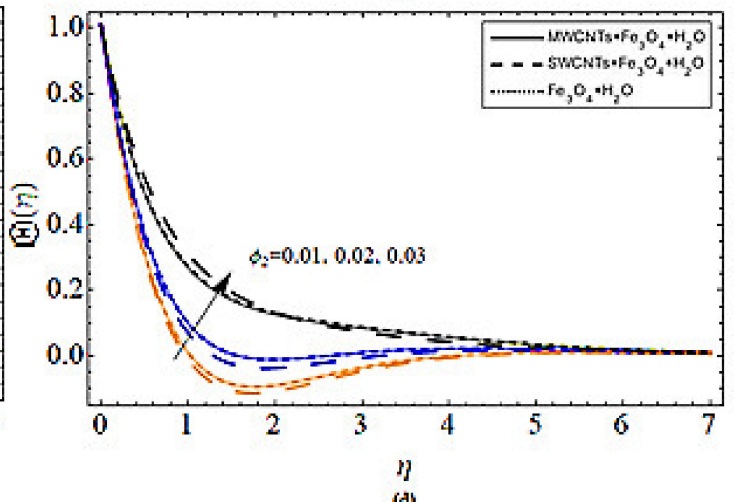

(d)

Figure 4. The behavior of energy profile $\Theta(\eta)$ versus (a) thermal energy ratio parameter $\gamma(\mathbf{b})$ Prandtl number $\operatorname{Pr}(\mathbf{c})$ volume friction parameter of CNTs $\phi_{1}(\mathbf{d})$ volume friction parameter of ferric oxide $\phi_{2}$. 


\subsection{Motile Microorganism and Concentration Profile}

Figure 5a-c manifested the behavior of motile microorganism $\Psi(\eta)$ and mass transfer profile $\Phi(\eta)$ versus the microorganism's distributions ratio $N_{\delta}$, bioconvective Peclet number $P e$ and Schmidt number $S, c$ respectively. The distributions of the microorganism's $N_{\delta}$ ration significantly enhances the motile microorganism profile as reported in Figure 5a. The increment in Peclet number Pe decreases the motile microorganism profile $\Psi(\eta)$ as shown in Figure $5 b$. The density of the microorganism reduces with the action of the Peclet number, which also decreases the motile microorganism profile. The consequences of the Schmidt number reduces the mass transition rate of hybrid nanofluid as revealed via Figure $5 \mathrm{c}$. The kinetic viscosity of viscous fluid flow improves with the action of Schmidt number Sc while the molecular diffusion rate diminishes, which results in the reduction of the mass propagation rate.
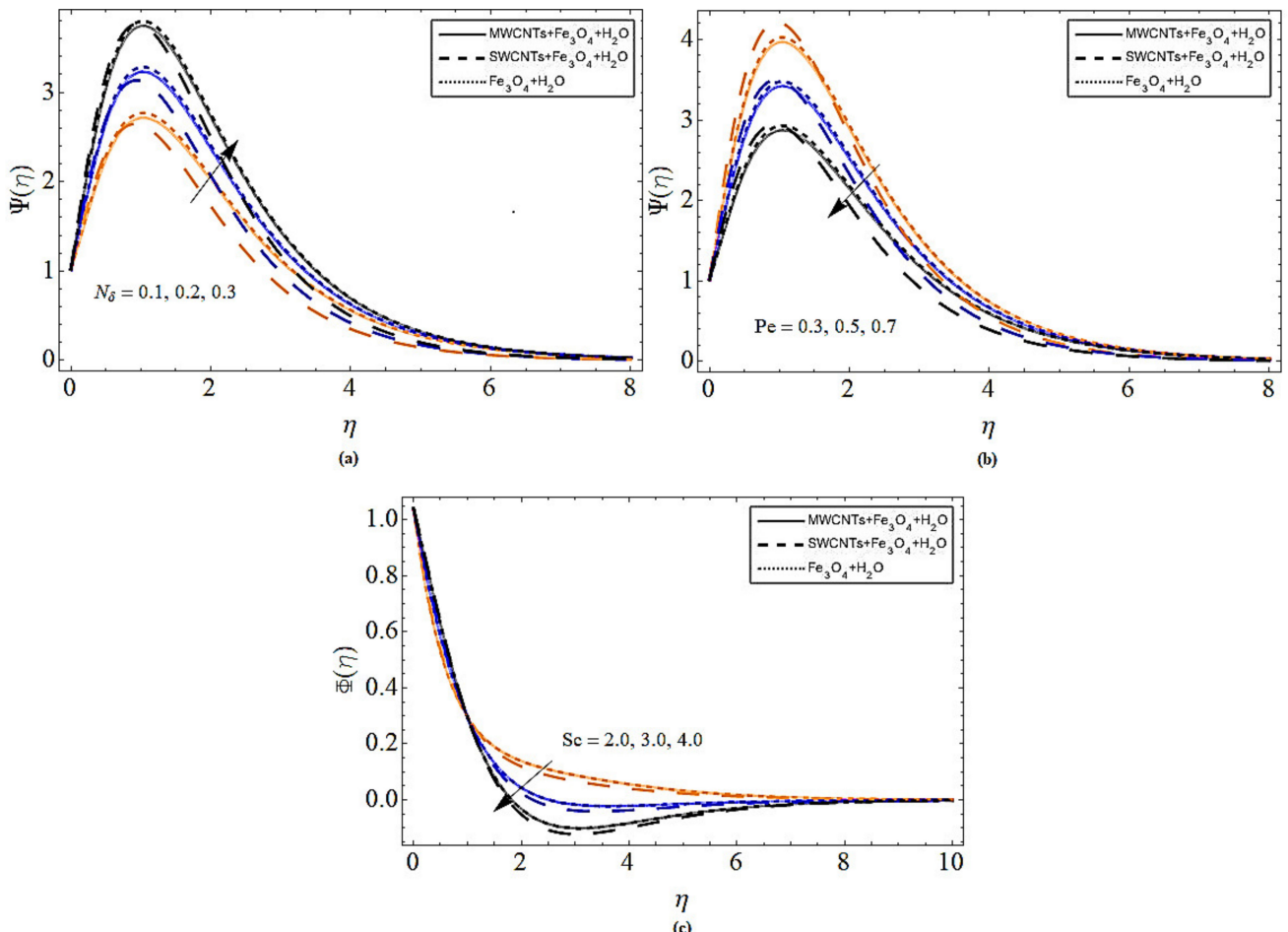

Figure 5. The behavior of motile microorganism $\Psi(\eta)$ and mass transfer profile $\Phi(\eta)$ versus (a) microorganisms distributions ratio $N_{\delta}$; (b) bioconvective Peclet number Pe; and (c) Schmidt number $S c$.

Table 3 addressed the numerical comparison of bvp4c versus the PCM method in order to ensure the validity of the present result. The axial velocity, radial velocity and temperature distribution are compared for this purpose. Tables 4 and 5 illustrate the comparative analysis for skin friction and the Nusselt number between magnetic nanoparticles and CNT's hybrid nanofluid.

Table 3. Comparative analysis between the bvp4c and PCM methods.

\begin{tabular}{ccccccc}
\hline \multicolumn{3}{c}{ bvp4c } & & \multicolumn{3}{c}{ PCM } \\
\hline$\eta$ & $f(\eta)$ & $g(\eta)$ & $\theta(\eta)$ & $f(\eta)$ & $g(\eta)$ & $\theta(\eta)$ \\
\hline 0.0 & 0.0000 & 0.0000 & 1.0000 & 0.0000 & 0.0000 & 1.0000 \\
0.4 & 0.0012 & 0.0030 & 0.1517 & 0.0011 & 0.0024 & 0.1421 \\
0.8 & 0.0070 & 0.0177 & 0.0932 & 0.00062 & 0.01086 & 0.0873 \\
1.2 & -0.0492 & -0.0871 & 0.0083 & -0.0453 & -0.0832 & 0.0071 \\
1.6 & -0.1458 & -0.2713 & 0.0039 & -0.1420 & -0.2709 & 0.0027 \\
\hline
\end{tabular}


Table 4. The comparative study of CNTs and ferric-oxide nanoparticles on the velocity field.

\begin{tabular}{ccccc}
\hline Title & \multicolumn{2}{c}{ CNTs } & \multicolumn{2}{c}{ Ferric Oxide $\mathrm{Fe}_{3} \boldsymbol{O}_{4}$} \\
\hline$\eta$ & $f^{\prime}(0)$ & $g^{\prime}(0)$ & $f^{\prime}(0)$ & $g^{\prime}(0)$ \\
\hline 0.00 & 1.2724 & 1.4912 & 1.3322 & 1.5562 \\
0.05 & 1.4133 & 1.5724 & 1.7734 & 1.6821 \\
0.01 & 1.6641 & 1.7054 & 2.0011 & 1.7922 \\
0.15 & 2.0300 & 2.0791 & 2.1701 & 1.1901 \\
0.20 & 2.1530 & 2.2753 & 2.3532 & 2.3102 \\
\hline
\end{tabular}

Table 5. Quantitive analysis for the Nusselt number and the Skin fraction.

\begin{tabular}{ccccccc}
\hline & \multicolumn{3}{c}{ CNTs } & \multicolumn{3}{c}{ Ferric Oxide $\boldsymbol{F e}_{3} \boldsymbol{O}_{4}$} \\
\hline$\eta$ & $h^{\prime}(0)$ & $\Theta^{\prime}(0)$ & $\Phi^{\prime}(0)$ & $h^{\prime}(0)$ & $\Theta^{\prime}(0)$ & $\Phi^{\prime}(0)$ \\
\hline 0.00 & 0.1724 & 1.5954 & 1.7953 & 0.2513 & 1.5581 & 1.6935 \\
0.04 & 0.1921 & 1.3362 & 1.6361 & 0.1835 & 1.6732 & 1.5316 \\
0.80 & 0.4139 & 1.1482 & 1.4481 & 0.3012 & 1.6621 & 1.3418 \\
0.12 & 0.2612 & 1.6014 & 1.2013 & 0.2410 & 2.2683 & 1.1031 \\
\hline
\end{tabular}

\section{Conclusions}

In this study, we presented the numerical approximation for microorganisms, CNTs, and ferric oxide water-based hybrid nanofluid flow induced by a wavy fluctuating spinning disc with energy propagation. The objective of the proposed analysis was to optimize thermal energy conveyance efficiency for a spectrum of industrial and biomedical applications. The phenomena have been expressed as a system of PDEs which are numerically computed while using the Matlab built-in package boundary value solver. The core findings from the above observations have been presented below:

- The inclusion of single- (SWCNTs) and multi-wall CNTs (MWCNTs) and magnetic nanoparticles $\phi_{2}=\phi_{\mathrm{Fe}_{2} \mathrm{O}_{3}}$ enhances the fluid velocity.

- The upward/downward fluctuation of the spinning disk encourages the fluid particles to move rapidly, which elevates the axial velocity of the fluid.

- The suction and injection effect over the surface of the spinning disk produces resistance to the flow field, which results in the reduction of the radial velocity $h(\eta)$ profile.

- The increment in disk rotation rate also excited the fluid particles during rotating, which resulted in rises in the radial velocity of fluid over a wavy surface.

- The thermal energy transmission rate declines with the impact of both thermal energy ratio parameter $\gamma$ and Prandtl number $P r$.

- The dispersion of nanomaterials in base fluid reduces the effectiveness of its heat absorption, which results in the enhancement of fluid temperature.

- The distributions of microorganism's $N_{\delta}$ ration significantly enhances the motile microorganism, while the increment in Peclet number Pe decreases the motile microorganism profile $\Psi(\eta)$.

- When compared to a homogeneous substrate, a wavy rotating surface elevates energy diffusion by as much as $15 \%$.

Author Contributions: Conceptualization, M.B. and A.S.; methodology, A.S.; software, T.G and W.K.; validation, I.A. and T.G.; formal analysis, A.S.; investigation, I.A.; data curation, M.B.; writing—original draft preparation, M.B.; writing—review and editing, M.B.; supervision, T.G.; project administration, P.K.; funding acquisition, P.K. All authors have read and agreed to the published version of the manuscript.

Funding: The financial support provided by the Center of Excellence in Theoretical and Computational Science (TaCS-CoE), KMUTT. Moreover, this research project is supported by Thailand Science Research and Innovation (TSRI) Basic Research Fund: Fiscal year 2021 under project number 64A306000005. 


\section{Institutional Review Board Statement: Not Applicable. \\ Informed Consent Statement: Not Applicable.}

Data Availability Statement: All the supporting data are within the manuscript.

Acknowledgments: The authors acknowledge the financial support provided by the Center of Excellence in Theoretical and Computational Science (TaCS-CoE), KMUTT. Moreover, this research project is supported by Thailand Science Research and Innovation (TSRI) Basic Research Fund: Fiscal year 2021 under project number 64A306000005.

Conflicts of Interest: The authors have no conflicts of interest.

\section{References}

1. Ahmadian, A.; Bilal, M.; Khan, M.A.; Asjad, M.I. The non-Newtonian maxwell nanofluid flow between two parallel rotating disks under the effects of magnetic field. Sci. Rep. 2020, 10, 1-14. [CrossRef]

2. Lv, Y.-P.; Algehyne, E.A.; Alshehri, M.G.; Alzahrani, E.; Bilal, M.; Khan, M.A.; Shuaib, M. Numerical approach towards gyrotactic microorganisms hybrid nanoliquid flow with the hall current and magnetic field over a spinning disk. Sci. Rep. 2021, 11, 1-13.

3. Li, Y.-X.; Muhammad, T.; Bilal, M.; Khan, M.A.; Ahmadian, A.; Pansera, B.A. Fractional simulation for Darcy-Forchheimer hybrid nanoliquid flow with partial slip over a spinning disk. Alex. Eng. J. 2021, 60, 4787-4796. [CrossRef]

4. Shuaib, M.; Shah, R.A.; Durrani, I.; Bilal, M. Electrokinetic viscous rotating disk flow of Poisson-Nernst-Planck equation for ion transport. J. Mol. Liq. 2020, 313, 113412. [CrossRef]

5. Shuaib, M.; Shah, R.A.; Bilal, M. Von-Karman rotating flow in variable magnetic field with variable physical properties. Adv. Mech. Eng. 2021. [CrossRef]

6. Shuaib, M.; Shah, R.A.; Bilal, M. Variable thickness flow over a rotating disk under the influence of variable magnetic field: An application to parametric continuation method. Adv. Mech. Eng. 2020. [CrossRef]

7. Hafeez, A.; Khan, M.; Ahmed, J. Thermal aspects of chemically reactive Oldroyd-B fluid flow over a rotating disk with CattaneoChristov heat flux theory. J. Therm. Anal. Calorim. 2021, 144, 793-803. [CrossRef]

8. Liang, M.; Fu, C.; Xiao, B.; Luo, L.; Wang, Z. A fractal study for the effective electrolyte diffusion through charged porous media. Int. J. Heat Mass Transf. 2019, 137, 365-371. [CrossRef]

9. Liang, M.; Liu, Y.; Xiao, B.; Yang, S.; Wang, Z.; Han, H. An analytical model for the transverse permeability of gas diffusion layer with electrical double layer effects in proton exchange membrane fuel cells. Int. J. Hydrog. Energy 2018, 43, 17880-17888. [CrossRef]

10. Jamil, F.; Ali, H.M. Applications of hybrid nanofluids in different fields. In Hybrid Nanofluids for Convection Heat Transfer; Elsevier: Amsterdam, The Netherlands, 2020; pp. 215-254.

11. Rao, Y. Nanofluids: Stability, phase diagram, rheology and applications. Particuology 2010, 8, 549-555. [CrossRef]

12. Fisher, C.; E Rider, A.; Jun Han, Z.; Kumar, S.; Levchenko, I.; Ostrikov, K.K. Applications and nanotoxicity of carbon nanotubes and graphene in biomedicine. J. Nanomater. 2012, 2012, 315185. [CrossRef]

13. Alawi, O.A.; Mallah, A.; Kazi, S.; Sidik, N.A.C.; Najafi, G. Thermophysical properties and stability of carbon nanostructures and metallic oxides nanofluids. J. Therm. Anal. Calorim. 2019, 135, 1545-1562. [CrossRef]

14. Gul, T.; Rahman, J.u.; Bilal, M.; Saeed, A.; Alghamdi, W.; Mukhtar, S.; Alrabaiah, H.; Bonyah, E. Viscous dissipated hybrid nanoliquid flow with Darcy-Forchheimer and forced convection over a moving thin needle. AIP Adv. 2020, 10, 105308. [CrossRef]

15. Tassaddiq, A.; Khan, S.; Bilal, M.; Gul, T.; Mukhtar, S.; Shah, Z.; Bonyah, E. Heat and mass transfer together with hybrid nanofluid flow over a rotating disk. AIP Adv. 2020, 10, 055317. [CrossRef]

16. Lim, A.E.; Lim, C.Y.; Lam, Y.C.; Taboryski, R. Electroosmotic flow in microchannel with black silicon nanostructures. Micromachines 2018, 9, 229. [CrossRef] [PubMed]

17. Lim, A.E.; Lam, Y.C. Numerical Investigation of Nanostructure Orientation on Electroosmotic Flow. Micromachines 2020, 11, 971. [CrossRef] [PubMed]

18. Bilal, M.; Khan, I.; Gul, T.; Tassaddiq, A.; Alghamdi, W.; Mukhtar, S.; Kumam, P. Darcy-forchheimer hybrid nano fluid flow with mixed convection past an inclined cylinder. CMC—Comput. Mater. Contin. 2021, 66, 2025-2039. [CrossRef]

19. Gul, T.; Bilal, M.; Shuaib, M.; Mukhtar, S.; Thounthong, P. Thin film flow of the water-based carbon nanotubes hybrid nanofluid under the magnetic effects. Heat Transf. 2020, 49, 3211-3227. [CrossRef]

20. Ghalandari, M.; Maleki, A.; Haghighi, A.; Shadloo, M.S.; Nazari, M.A.; Tlili, I. Applications of nanofluids containing carbon nanotubes in solar energy systems: A review. J. Mol. Liq. 2020, 313, 113476. [CrossRef]

21. Waqas, H.; Imran, M.; Bhatti, M. Influence of bioconvection on Maxwell nanofluid flow with the swimming of motile microorganisms over a vertical rotating cylinder. Chin. J. Phys. 2020, 68, 558-577. [CrossRef]

22. Khan, M.I.; Khan, S.U.; Jameel, M.; Chu, Y.-M.; Tlili, I.; Kadry, S. Significance of temperature-dependent viscosity and thermal conductivity of Walter's B nanoliquid when sinusodal wall and motile microorganisms density are significant. Surf. Interfaces 2021, 22, 100849. [CrossRef]

23. Kotha, G.; Kolipaula, V.R.; Rao, M.V.S.; Penki, S.; Chamkha, A.J. Internal heat generation on bioconvection of an MHD nanofluid flow due to gyrotactic microorganisms. Eur. Phys. J. Plus 2020, 135, 600. [CrossRef] 
24. Ahmadian, A.; Bilal, M.; Khan, M.A.; Asjad, M.I. Numerical analysis of thermal conductive hybrid nanofluid flow over the surface of a wavy spinning disk. Sci. Rep. 2020, 10, 18776. [CrossRef] [PubMed]

25. Hussain, T.; Xu, H.; Raees, A.; Zhao, Q.-K. Unsteady three-dimensional MHD flow and heat transfer in porous medium suspended with both microorganisms and nanoparticles due to rotating disks. J. Therm. Anal. Calorim. 2021. [CrossRef]

26. Ma, Y.; Mohebbi, R.; Rashidi, M.; Yang, Z. MHD convective heat transfer of Ag-MgO/water hybrid nanofluid in a channel with active heaters and coolers. Int. J. Heat Mass Transf. 2019, 137, 714-726. [CrossRef]

27. Bilal, M.; Gul, T.; Alsubie, A.; Ali, I. Axisymmetric hybrid nanofluid flow with heat and mass transfer amongst the two gyrating plates. ZAMM J. Appl. Math. Mech. Z. Angew. Math. Mech. 2021. [CrossRef]

28. Turkyilmazoglu, M. Fluid flow and heat transfer over a rotating and vertically moving disk. Phys. Fluids 2018, $30,063605$. [CrossRef] 